\title{
Clustering of galaxies in the overdense regions of radio galaxies at $z>0.6$
}

\author{
Nedelia A. Popescu \\ Astronomical Institute of Romanian Academy, Bucharest, RO-040557, Romania \\ email: nedelia@aira.astro.ro
}

\begin{abstract}
Photometric redshifts technique and red sequence technique are used in order to analyze the clustering of galaxies in the environments of 4 radio galaxies with redshifts $z>0.6$.
\end{abstract}

Keywords. galaxies: photometry,galaxies: distances and redshifts, galaxies: clusters

The optical and near infrared photometric data, completed with HST morphological data, for radio galaxies 3C220.1 $(z=0.62)$, 3C34 $(z=0.689), 3 \mathrm{C} 184(z=0.996)$ and 3C210 ( $z=1.169$ ) are considered (Stanford et al. 2002). The primary aims of this paper are to determine the photometric redshifts for the studied galaxies and to search clustering features of galaxies with similar redshifts. The photometric redshifts technique (Z-PEG software) is used in order to analyze the clustering of galaxies in the environments of these radio galaxies. The comparison of the HST morphology of galaxies with the model spectral galaxy type (determined with Z-PEG software - Le Borgne \& RoccaVolmerange 2002) is in a good agreement, confirming the importance of the photometric redshifts determinations. The clustering features for galaxies with similar redshifts are revealed in the field of 3C220.1 (see figure 1), 3C34 and 3C210 (Popescu 2006).
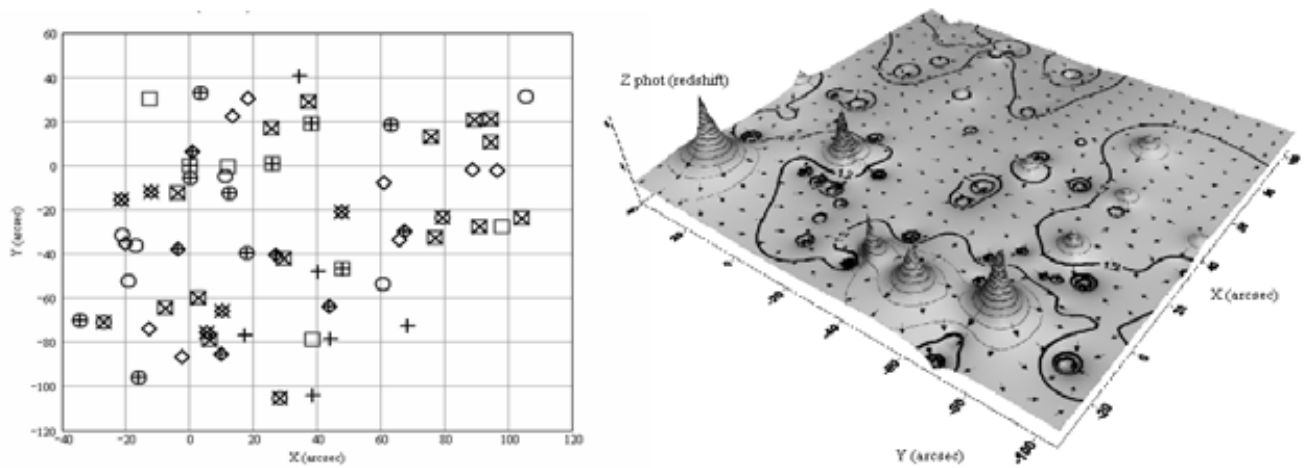

Figure 1. 2D distribution of galaxies function of morphology (sqaures $-\mathrm{E} / \mathrm{S} 0$; circles $-\mathrm{S}$; diamonds - Irr, mergers; $(+)-17$ gal. with $0.45<z<0.7,(\mathrm{X})-26$ gal. with $0.75<z<1.05$ clustered in three groups, containing $15 \mathrm{E} / \mathrm{S} 0$ ) (left) and 3D distribution of galaxies (right)

\section{References}

Stanford, S. A., Eisenhardt, P., Dickinson, M., Holden, B., \& De Propris, R. 2002, ApJS 142, 153.

Le Borgne, D. \& Rocca-Volmerange, B. 2002, A\&A A 386, 446.

Popescu, N. A. 2006, Rom.Astron.J.S. 16, 135. 